livraisons

d'Histoire

de l'Architecture

\section{Livraisons de l'histoire de l'architecture}

19 | 2010

Du remploi à l'éphémère

\title{
L'administration des cultes et le financement des pontificaux sous le Second Empire
}

The Administration des Cultes and the pontificals' funding during the Second Empire

Kirchenverwaltung und staatliche Finanzierung von festlichen Messgewändern im Second Empire

\section{Maria-Anne Privat-Savigny}

\section{OpenEdition}

\section{Journals}

Édition électronique

URL : http://journals.openedition.org/lha/242

DOI : $10.4000 /$ lha. 242

ISSN : 1960-5994

Éditeur

Association Livraisons d'histoire de l'architecture - LHA

Édition imprimée

Date de publication : 10 juin 2010

Pagination : $97-110$

ISSN : 1627-4970

Référence électronique

Maria-Anne Privat-Savigny, « L'administration des cultes et le financement des pontificaux sous le Second Empire », Livraisons de l'histoire de l'architecture [En ligne], 19 | 2010, mis en ligne le 10 juin 2012, consulté le 30 avril 2019. URL : http://journals.openedition.org//ha/242 ; DOI : 10.4000/Iha.242

Ce document a été généré automatiquement le 30 avril 2019

Tous droits réservés à l'Association LHA 


\section{L'administration des cultes et le} financement des pontificaux sous le Second Empire

The Administration des Cultes and the pontificals' funding during the Second Empire

Kirchenverwaltung und staatliche Finanzierung von festlichen Messgewändern im Second Empire

\section{Maria-Anne Privat-Savigny}

1 En 1978 dans la Revue des monuments historiques, Jean-Michel Leniaud publiait une étude fondatrice, consacrée au trésor liturgique de la cathédrale de Moulins. En étudiant les pontificaux ayant appartenu à Mgr de Dreux-Brézé, il effectuait un rapprochement convaincant entre le pontifical blanc de l'évêque de Moulins et un devis découvert dans les fonds des Cultes aux Archives nationales, devis établi par Hubert Ménage en 1852, mettant en exergue un financement public effectué par l'administration des cultes pour la confection de pontificaux. Une quinzaine d'années plus tard, Élisabeth Hardouin-Fugier publiait un premier sondage de ces aides publiques ${ }^{1}$.

2 L'importante commande de 1852 et le financement public conséquent accordé au bénéfice d'une fabrique et d'un évêque, qui n'était pourtant pas des plus favorables à Napoléon III, suscitent des interrogations sur la politique menée par l'administration des cultes sous le Second Empire pour l'achat de pontificaux. Si, dans le domaine administratif, elle n'a guère innové, pérennisant les choix du passé, elle a accordé une somme importante au financement de pontificaux, faisant face à la nécessité de renouveler le vestiaire liturgique. L'analyse de l'évolution des sommes octroyées nous fait nous interroger sur la répartition géographique et chronologique de ces aides publiques, mais aussi sur leur éventuel rôle politique, voire religieux; peut-on par exemple y voir l'évolution des influences gallicanes et ultramontaines? Ou d'une corrélation entre les montants accordés et la politique religieuse de Napoléon III, voire l'évolution de ses relations avec 
le Saint-Siège? Certes, le pontifical, à travers les financements publics, est incontestablement un outil politique, il n'en demeure pas moins un objet esthétique et l'administration des cultes pendant le Second Empire, mais s'efforce d'entreprendre une politique stylistique engagée, son bras armé étant la commission des arts et édifices religieux mise en place à la fin des années 1840 . Peut-on pour autant parler d'un style propre au Second Empire dans ce domaine?

\section{Le pontifical et les procédures administratives sous le Second Empire}

3 D'un point de vue administratif, le Second Empire n'innove guère quant à la réglementation relative aux financements publics des pontificaux. Celle-ci s'est mise en place progressivement, surtout après la réorganisation de l'administration des Cultes dans les années $1840^{2}$, même si un certain nombre de circulaires ministérielles antérieures avaient déjà établi l'essentiel de la procédure. Le texte qui toutefois demeure une référence pendant le Concordat est la circulaire ministérielle du 20 décembre 1841, qui pose des principes durables. Elle ne traite que des dépenses relatives au chapitre $9 \mathrm{du}$ budget des cultes lequel concerne les dépenses du service intérieur des édifices diocésains, dont "le secours aux fabriques pour achats d'ornements pontificaux». Elle fixe également les modalités de la procédure à suivre et les documents justificatifs à fournir au dossier pour que ce dernier soit examiné par l'administration ${ }^{3}$. Plusieurs textes viennent la compléter notamment en 1843, 1847 et 1848, donc principalement sous la Monarchie de Juillet. Le Second Empire n'est pas revenu sur les principes administratifs établis par les gouvernements précédents, mais en a poursuivi l'application avec une certaine rigueur, refusant de traiter tout dossier ne répondant pas aux règles définies par ces textes. Le $1^{\text {er }}$ août 1863 par exemple, l'évêque de Nancy se voit retourner sa demande de financement parce que c'est à la fabrique de la cathédrale et non au prélat de la formuler, même si dans les faits l'évêque joue un rôle majeur ${ }^{4}$. De même en 1855 , le règlement d'un acompte au lyonnais Ribolet-Bauchu pour des travaux réalisés pour la fabrique de la cathédrale d'Ajaccio est rejeté car ce dernier aurait refusé de signer le certificat d'avancée des travaux, rédigé par le trésorier de la fabrique ${ }^{5}$. Le paiement du solde de 6800 francs se trouve aussi compromis par l'administration des Cultes, car Ribolet-Bauchu n'a ni certifié ni quittancé sa facture, entraînant un retard notable de paiement ${ }^{6}$.

Toutefois, l'analyse des délais dans le traitement des dossiers, montre que l'administration des Cultes sous le Second Empire se montre plutôt efficace, le délai de réponse étant réduit surtout lorsqu'une visite du couple impérial est annoncée. Ainsi à Marseille en 1852, il n'a fallu que dix jours pour valider une dépense de 5 192,20 francs et à peine plus pour que l'administration des Cultes en accepte un important dépassement ${ }^{7}$. Une situation similaire se reproduit à Moulins en 1853, au Mans en 1869, à Ajaccio en 1868.

5 Si l'administration des Cultes répond dans les délais, elle répond parfois négativement. Ses refus sont toutefois peu nombreux. Seulement $27,4 \%$ de la totalité des refus recensés pendant le Concordat, le sont sous le Second Empire. Mais ils ne représentent que 6,63\% du total des dossiers soumis à l'administration des Cultes pendant les années impériales et $6,6 \%$ du total des sommes demandées (ill. 1). 
III. 1 : Graphique montrant le différentiel existant entre les sommes allouées et les sommes demandées pour l'achat de pontificaux par les fabriques des cathédrales à l'administration des Cultes. Évolution pendant le Second Empire.

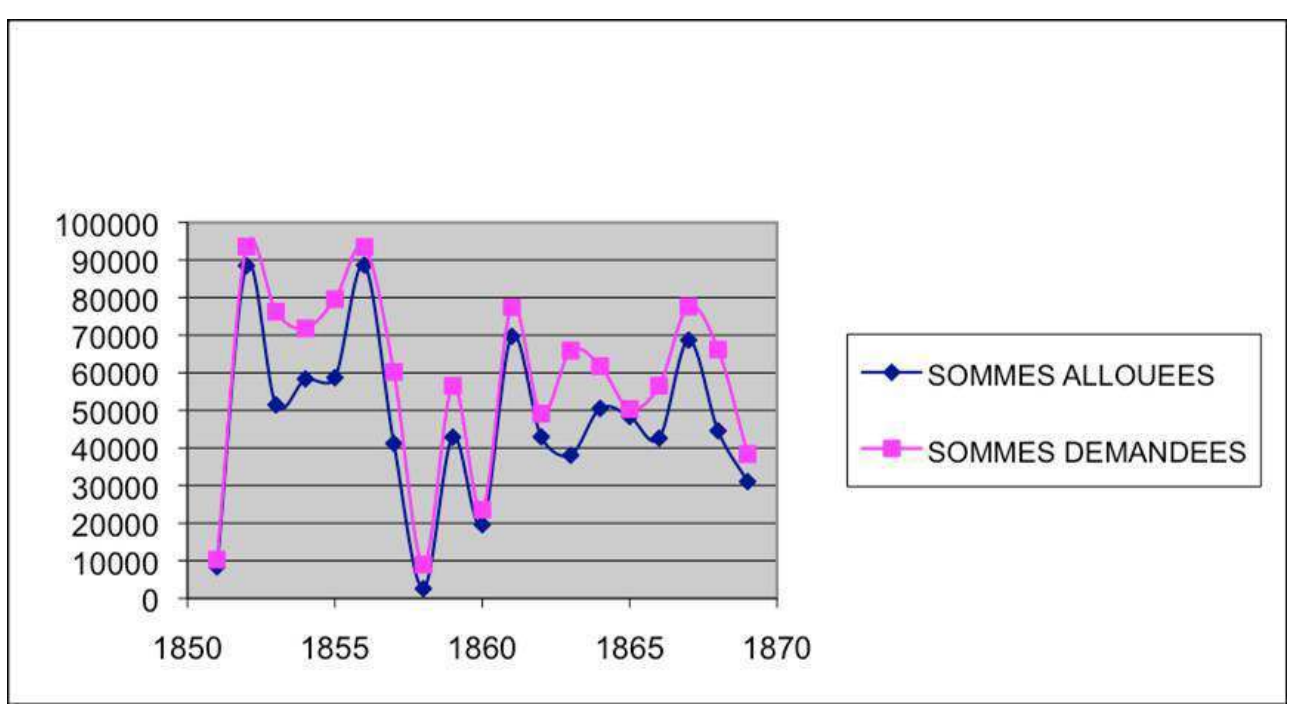

6 Les refus datent majoritairement des années 1850 et sont formulés par Fortoul et Rouland. Ils ont deux origines principales : le montant élevé des requêtes qui excèdent 10000 francs d'une part et d'autre part, le traitement du dossier par la commission des arts et édifices religieux, qui aboutit, comme à Blois en 1853 à un refus, quand un désaccord important apparaît entre la fabrique, l'évêque et les membres de cette commission ${ }^{8}$.

7 Quand le dossier est accepté, l'administration des Cultes le finance soit en totalité soit partiellement. Si la majorité des pontificaux est payée sur une seule année, on observe une multiplication des dossiers financés sur plusieurs exercices. Leur analyse montre que, sans doute en raison de l'abondance des demandes, le financement pluriannuel permet de répondre favorablement à toutes les requêtes formulées par les fabriques. Ce phénomène est particulièrement significatif dans les années 1856,1857 et 1858, même pour les devis de faible montant et aucune règle ne semble présider à cette prise de décision. L'analyse globale du budget des cultes montre que $43 \%$ du montant total alloué pendant les années concordataires l'a été au cours du Second Empire : 896801 francs ont ainsi été consacrés à ce type de dépenses, alors que le total des demandes atteint 1117606 francs (ill. 2). On note d'ailleurs un bon taux de financement des dossiers soumis à l'approbation de l'administration des Cultes, même si des variations notables sont à souligner. 
III. 2 : Graphique montrant la répartition des financements alloués à l'achat de pontificaux par l'administration des Cultes au cours de la période concordataire.

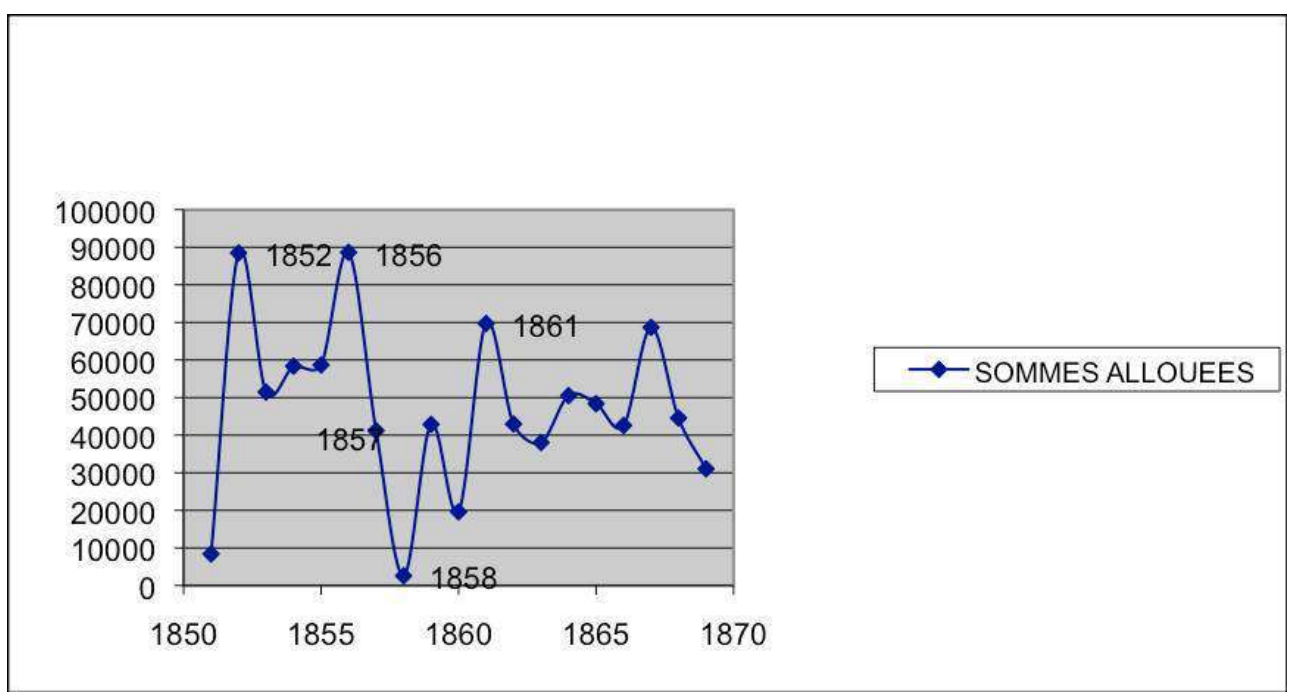

8 La répartition des crédits entre les trois principaux ministres des Cultes de la période impériale montre une certaine égalité : Fortoul est à l'origine de $39 \%$ des allocations, Baroche, de $31 \%$, Rouland, de $28 \%$. Deux cent quarante et un dossiers ont été déposés auprès de l'administration sous le Second Empire, soit une moyenne de trois dossiers par diocèse. La moyenne des sommes allouées par cathédrale est de 12617 francs, ce qui est assez conséquent; le montant moyen des demandes est de 15741 francs, tandis que le montant moyen obtenu est de 12741 francs. On observe également que 35,2\% des dossiers acceptés présentent un coût supérieur à la moyenne. Les fabriques les mieux dotées pendant la période étudiée sont celles de Toulouse (46461 francs, soit $5 \%$ du total), d'Annecy (41 900 francs), de Besançon (40 027 francs), de Tours (35028 francs, uniquement pour trois commandes), d'Ajaccio (31 425 francs) (ill. 3). 
III. 3 : Graphique montrant la répartition géographique des subventions allouées par l'administration des Cultes pour l'achat de pontificaux pendant le Second Empire.

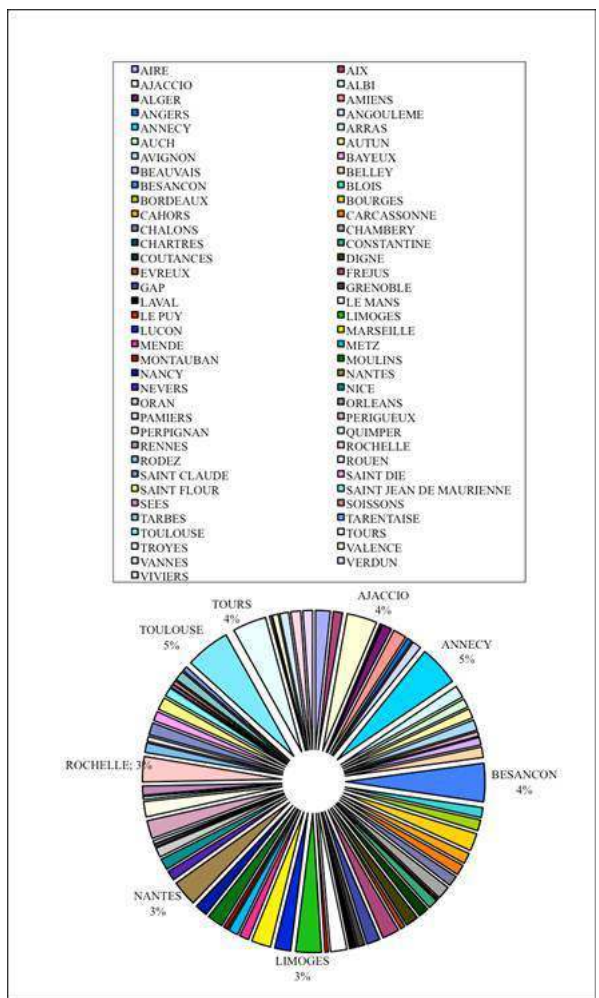

9 Les budgets alloués à l'achat de pontificaux atteignent leur apogée en 1852 et 1856, puis ils chutent en 1857 et connaissent un abîme en 1858 ; ils renaissent les années suivantes, notamment en 1861 et 1868 , sans toutefois atteindre les niveaux des premières années du Second Empire (ill. 4).

III. 4 : Graphique montrant l'évolution du budget alloué par l'administration des Cultes à l'achat de pontificaux au cours du Second Empire.

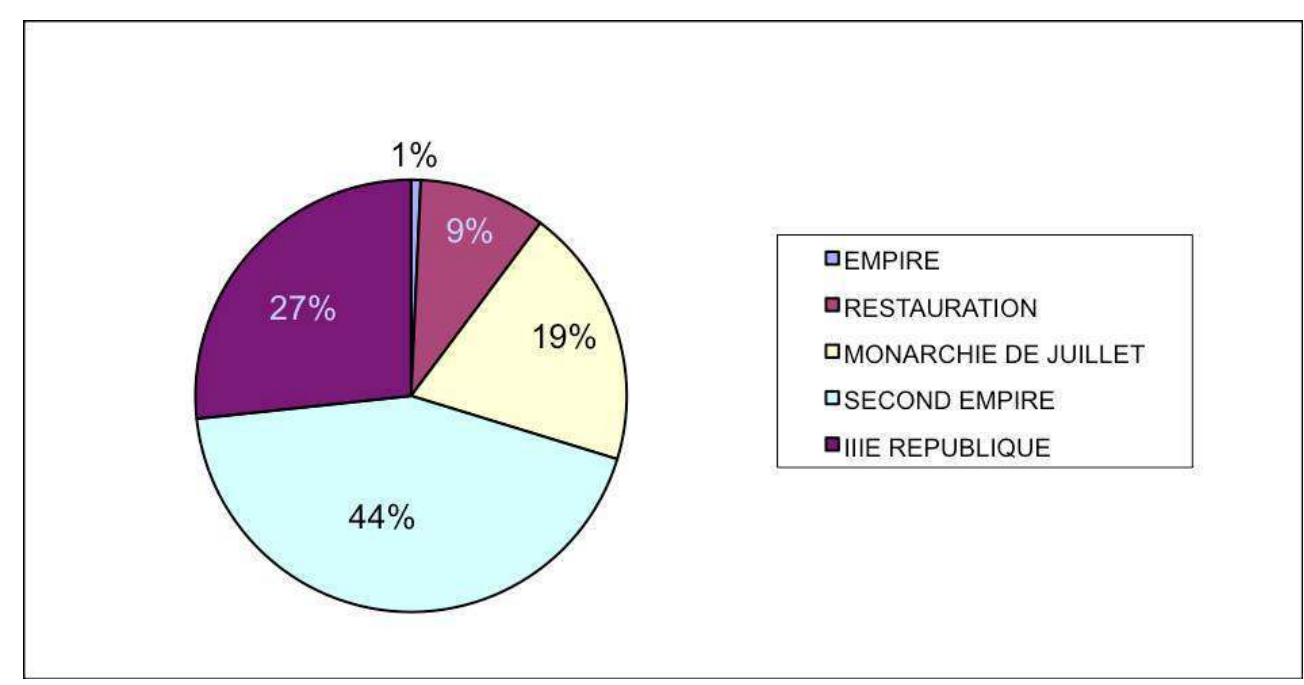

10 Faut-il voir dans cette évolution un impact de la politique religieuse de Napoléon III ou plutôt de ses relations avec le Pape, ou encore de sa politique italienne? Le pontifical, surtout quand il est confectionné en drap d'or ou en drap d'argent, n'est-il pas le reflet 
immédiat de la politique gouvernementale en faveur de l'Église, symbolisant par là-même l'harmonie des relations entre l'État et l'Église? Il n'est pas anodin de constater précisément à partir de 1857, quand les relations entre Napoléon III et la papauté commencent à s'assombrir, une chute importante des crédits alloués à l'achat de pontificaux.

\section{Le pontifical : un outil politique}

Il est assez aisé d'établir une corrélation entre l'octroi d'une subvention et la personnalité du prélat en place. Par exemple, la requête d'un montant de 8763,95 francs formulée en 1852 par Mgr Villecourt, ultramontain intransigeant sera bloquée jusqu'à l'avènement de Mgr Landriot en 1855, proche du gouvernement, libéral et plus tolérant envers les protestants que son prédécesseur' ${ }^{9}$. D'une manière générale, on note que les fabriques qui ont bénéficié d'une aide supérieure à la moyenne ont un évêque proche ou dévoué au gouvernement impérial. Au Mans, les aides financières sont accordées après la mort du gallican tumultueux Mgr Bouvier, à qui son successeur, Mgr Nanquette, ultramontain et prévenant envers la cause impériale bénéficiera des largesses de l'administration ${ }^{10}$. À Luçon, le rôle apaisant de Mgr Delamarre, également proche du gouvernement impérial, qui succède au bouillant Mgr Baillès, lui permet d'obtenir le financement d'un pontifical en drap d'or fin brodé d'or fin par Henry Lemoine de Nantes pour un coût de 11 055,60 francs ${ }^{11}$. Les exemples sont fort nombreux : citons Mgr Dépery, évêque de Gap, favorable à l'Empire et qui bénéficie de nombreuses subventions ${ }^{12}$ ou encore Mgr Salinis, évêque ultramontain d'Amiens mais rallié à Napoléon III comme son successeur Mgr Boudinet, qui arrive à Amiens en 1856 et qui reçoit de nombreux financements publics pour la confection de pontificaux ${ }^{13}$. Force est de constater que, dans de nombreux dossiers, le préfet ou l'administration vérifient avant l'acceptation d'un dossier si le prélat fait chanter le Domine Salvum en l'honneur de la famille impériale, argument souvent décisif. Force est aussi de remarquer que les tendances plutôt gallicanes ou plutôt ultramontaines des prélats apparaissent cependant secondaires dans la politique menée par l'administration des Cultes concernant les pontificaux. Les prélats nommés par Fortoul, Rouland ou Baroche bénéficient presqu'automatiquement de financements publics quelle que soit leur tendance. On note par ailleurs la mise en place d'une politique précise en faveur des diocèses devenus français, en Afrique du Nord, en Algérie surtout, en Savoie et à Nice. Baroche s'occupe des cathédrales d'Algérie et octroie à la fabrique de la cathédrale d'Alger une somme totale de 10000 francs pour l'achat de pontificaux en 1866 et en $1869^{14}$ , somme élevée, mais en deçà de la moyenne octroyée par l'administration pendant la période impériale. Rouland entreprend une politique active en faveur des diocèses de Savoie, et particulièrement de la fabrique de la cathédrale d'Annecy, surtout entre 1861 et 1862.

Le Second Empire ne fait que poursuivre une habitude engagée pendant la Restauration et la Monarchie de Juillet, faisant du pontifical un outil politique. Toutefois, contrairement à Louis-Philippe qui a financé de nombreux pontificaux pour les fabriques des cathédrales sur les fonds de la liste civile, Napoléon III a pris, sur ce budget plus personnel, une autre option, privilégiant les petites églises sur l'ensemble du territoire ${ }^{15}$.

Outil politique, le pontifical devient surtout au cours du Second Empire un objet esthétique. Peut-on caractériser un style propre au Second Empire dans la réalisation des 
pontificaux financés par l'administration des Cultes? Quelle position l'administration des Cultes a-t-elle adoptée par rapport au courant néogothique pour ce type de production?

\section{Le pontifical et la politique artistique de l'administration des Cultes sous le Second Empire}

L'analyse par couleur liturgique des devis soumis à l'approbation de l'administration des Cultes, permet de dégager quelques tendances notamment dans le choix des tissus. Ainsi, pour les pontificaux de couleur verte, le damas broché or fin est particulièrement apprécié. Le velours et la moire antique sont plutôt choisis pour les pontificaux de couleur violette, comme la chasuble et ses accessoires en moire antique fournis par Roulin pour la fabrique de la cathédrale de Verdun en 1866 pour un montant de 650 francs, ce qui est relativement conséquent ${ }^{16}$. Citons également le pontifical livré par Ribolet-Bauchu en 1854 à la fabrique de la cathédrale d'Ajaccio, également en moire antique, mais d'une qualité supérieure, le prix de la chasuble se montant à 800 francs $^{17}$. Pour les pontificaux de couleur blanche, le damas broché or fin, qui au demeurant domine toute la période concordataire, est aussi fort apprécié pendant la période impériale. Mentionnons notamment le pontifical blanc fourni par Girerd frères de Lyon pour la fabrique de la cathédrale Saint-Jean-de-Maurienne en $1861^{18}$. Si la majorité des pontificaux blancs (hors drap d'argent) sont relativement peu onéreux, le prix de la chasuble se situant entre 220 et 700 francs, les pontificaux blancs réalisés sous le Second Empire font exception et se distinguent par leur grande qualité. Ainsi le pontifical en moire antique brodé d'or fin livré par Quinet Limal Boutron en 1864 à la fabrique de la cathédrale de Nevers (ill.5), se compose d'une chasuble à 750 francs et d'une chape à 1200 francs $^{19}$. 
III. 5 : Quinet Limal-Boutron, Pontifical en moire antique blanche brodé de filés métalliques dorés et de soies polychromes, réalisé pour la fabrique de la cathédrale de Nevers, 1864

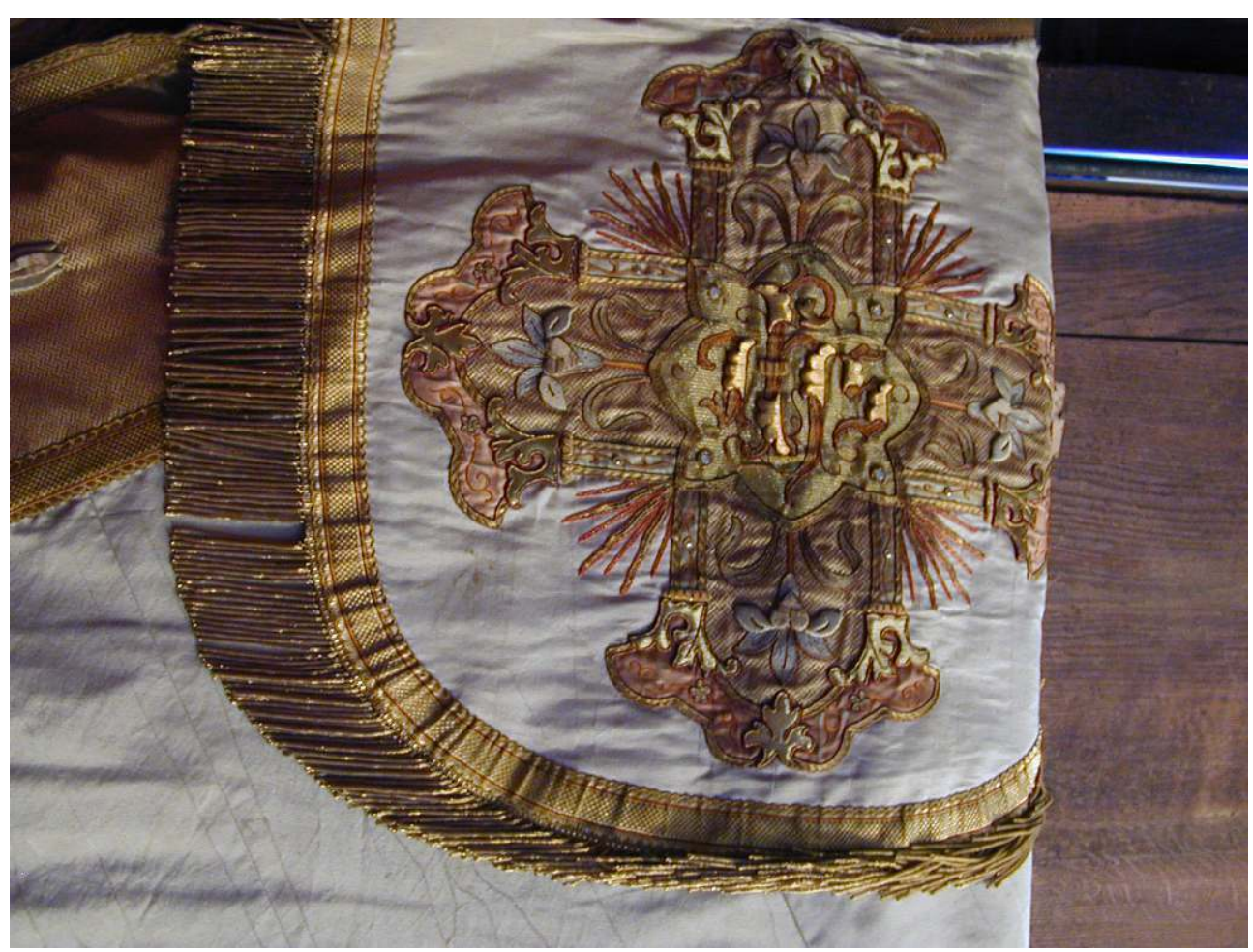

NEVERS, SACRISTIE DE LA CATHÉDRALE

CL. M.-A. P.-S.

15 La broderie d'or et de soies polychromes est particulièrement riche, comme celle de la chasuble réalisée par la maison Henry pour la fabrique de la cathédrale d'Ajaccio en 1868, en moire antique entièrement brodée d'or fin, de soies et en relief ${ }^{20}$. Plusieurs pontificaux de forme dite moyen âge appartiennent à cette catégorie et présentent une qualité supérieure. Le pontifical en "brocart de soie blanc" livré par Hubert Ménage à la fabrique de la cathédrale du Mans en 1865, est richement brodé en applications (ill. 6). 


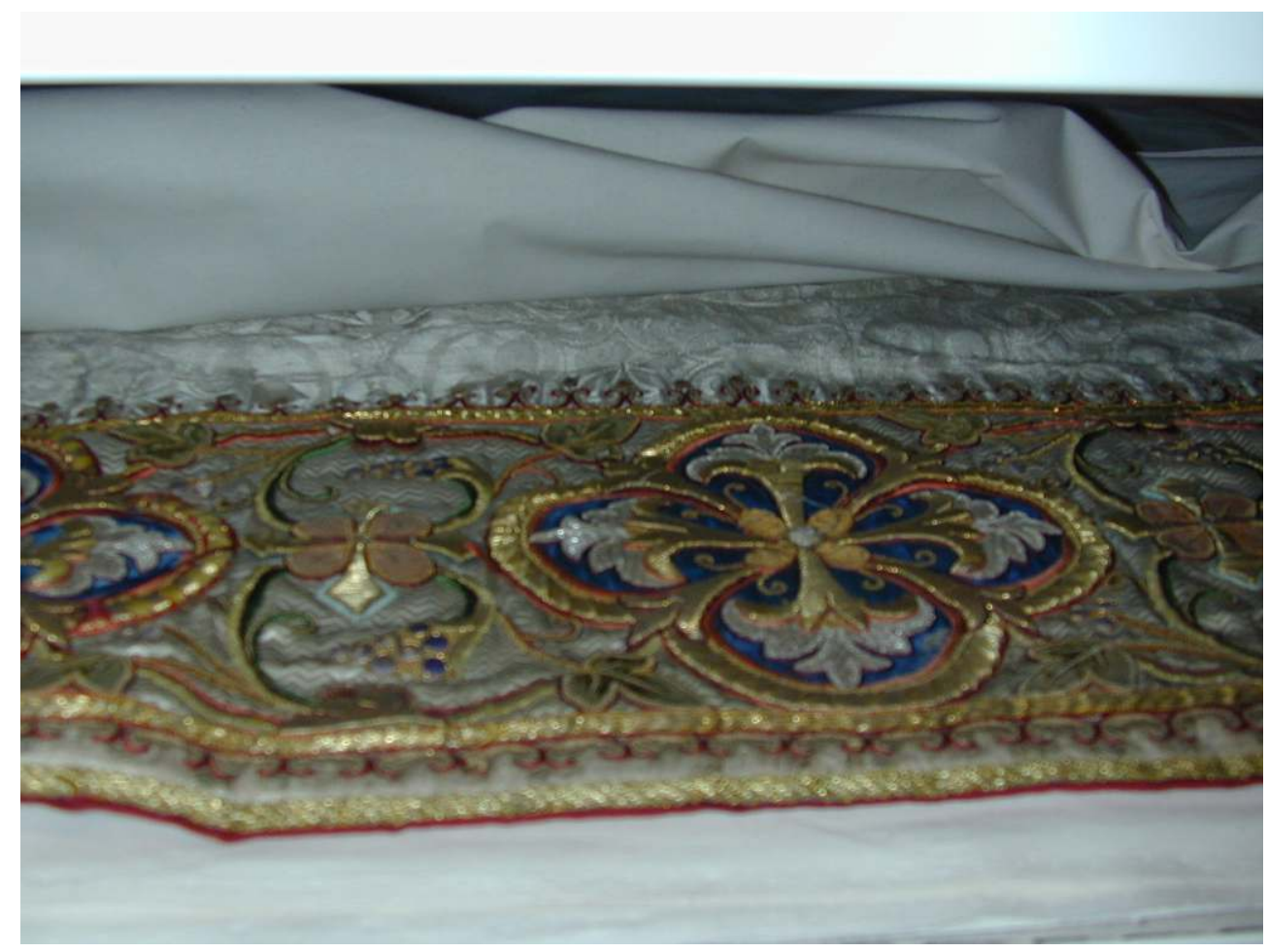

LE MANS, CONSERVATION DES ANTIQUITÉS ET OBJETS D'ART

CL. M.-A. P.-S.

16 Le prix de la chasuble atteint 1000 francs, ce qui est considérable ${ }^{21}$. Pour les pontificaux de couleur rouge, la forme romaine domine largement, comme le velours de soie. Le Second Empire se distingue néanmoins par la fourniture de plusieurs pontificaux rouges de très haute qualité, comme celui livré par Hubert Ménage à la fabrique de la cathédrale $\mathrm{du}$ Mans en $1869^{22}$. Les pontificaux noirs sont majoritairement en velours de soie et présentent, même sous le Second Empire, une grande standardisation que ce soit dans la forme, ils sont tous de forme romaine, ou dans le décor, généralement constitué d'applications de broderies sur carton en filés métalliques dorés ou argentés. Toutefois, une nouvelle fois le Second Empire se différencie par quelques réalisations de qualité supérieure, comme ce pontifical en moire antique noire brodée d'or fin réalisé pour la fabrique de la cathédrale de Toulouse en 1861. Le prix de la chasuble se monte à 800 francs $^{23}$. Mais si un style propre au Second Empire devait être défini, il se rapporterait aux pontificaux en drap d'or ou en drap d'argent surchargés de broderies d'or et d'argent. Bien que relativement stéréotypés, ces pontificaux présentent non seulement une grande qualité, mais aussi un coût fort élevé, situé en moyenne entre 10000 francs et 15000 francs. Citons par exemple le pontifical en drap d'argent livré par Dubus à la fabrique de la cathédrale de Toulouse en 1854 pour la somme de 18000 francs, l'administration des Cultes ne finançant que 12000 francs de cette dépense considérable ${ }^{24}$. Le drap d'argent est broché et « coloré en broderie tout or fin ». Le pontifical commandé par Mgr Boudinet pour la fabrique de la cathédrale d'Amiens en 1867 appartient aussi à cette catégorie. Il est confié aux religieuses de la Trappe Notre-Dame-de-Toute-Consolation de Lyon Vaise pour la somme de 15000 francs, l'administration des Cultes n'en payant que les deuxtiers. La broderie est étonnamment riche, réalisée sur un fond d'or en peinture à 
l'aiguille, tandis que le tissu est un drap d'argent broché or fin dessinant des motifs variés notamment d'épis de blé et de grappes de raisin. Les pontificaux en drap d'or présentent une grande diversité. Toutefois, le Second Empire a su produire les plus beaux exemples, comme le pontifical fourni par Biais Aîné et $C^{\text {ie }}$ à la fabrique de la cathédrale de Périgueux en 1863. Le prix de la chasuble atteint 1300 francs. Il s'agit « d'une chasuble en drap d'or fin brodée or fin rehaussée de soies de couleurs, garnie d'or, doublée de soie ${ }^{25}$. Quatre années plus tard, en 1867, la même fabrique reçoit un autre pontifical également en drap d'or livré par Dupuis d'un montant total de 9500 francs; la chasuble vaut 800 francs, la chape coûte 1000 francs, la qualité demeure élevée ${ }^{26}$. Les broderies polychromes montrent combien le talent des brodeurs n'a pas été perdu. Le pontifical commandé par Mgr Boudinet pour la fabrique de la cathédrale d'Amiens en 1867 à A. Favier Neveux ${ }^{27}$, orfèvre rue de l'Archevêché à Lyon, l'illustre (ill. 7 et 8).

III. 7 : A. Favier, Chasuble du pontifical en drap d'or réalisé pour la fabrique de la cathédrale d'Amiens en 1867

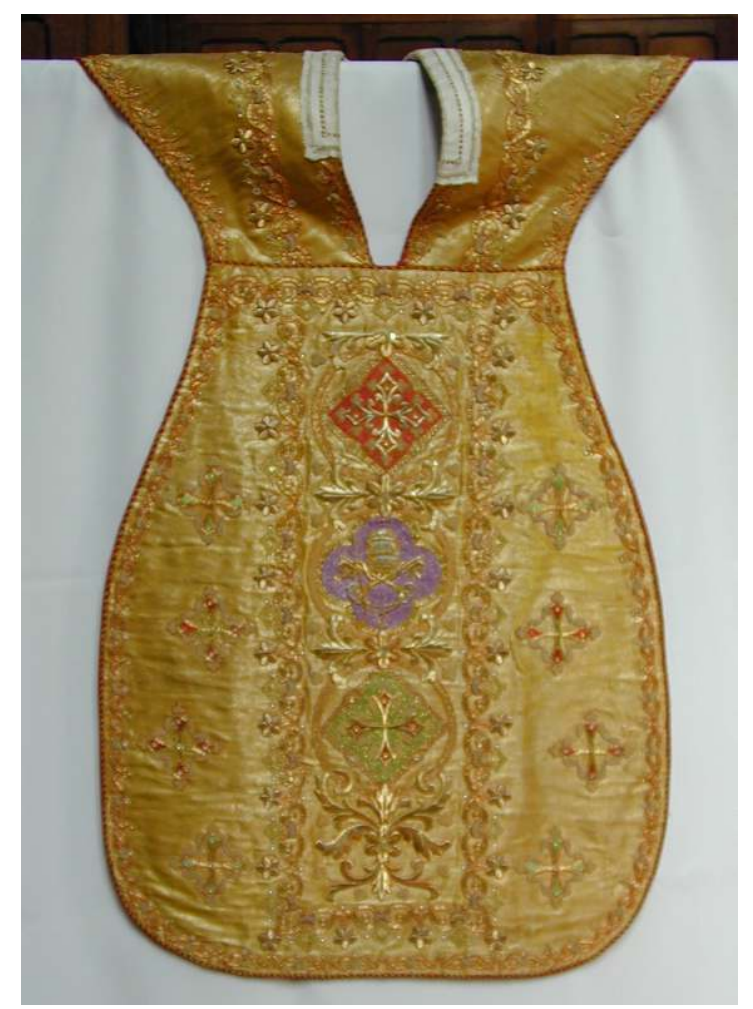

AMIENS, SACRISTIE DE LA CATHÉDRALE

CL. M.-A. P.-S. 
III. 8 : A. Favier, Dos de la chasuble du pontifical en drap d'or réalisé pour la fabrique de la cathédrale d'Amiens en 1867, détail du centre

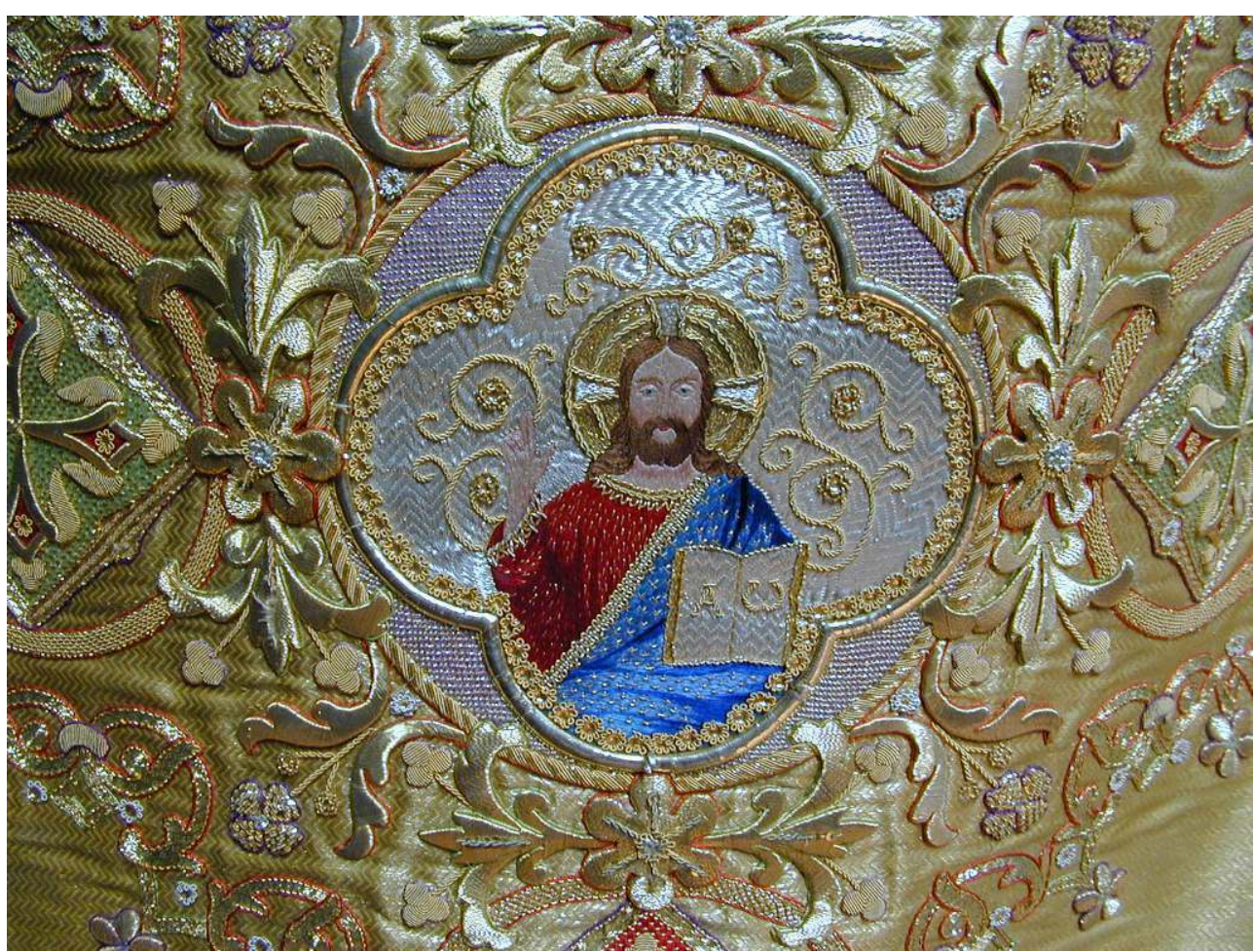

AMIENS, SACRISTIE DE LA CATHÉDRALE

CL. M.-A. P.-S.

Ce pontifical en drap d'or fin d'un montant de 15000 francs, est encore complet et est enrichi de broderies réalisées par les religieuses trappistes de Lyon, dont les modèles sont dans l'Album de broderies religieuses, édité par le Père Martin en 1854, album qui connaît un succès croissant à partir du milieu des années 1860 , montrant l'influence du courant néogothique.

18 Le R.-P. Martin figure parmi les membres de la section «vitraux et ornements » de la commission des arts et édifices religieux, sorte de bras armé de l'administration des Cultes pour l'établissement d'une politique stylistique. Il compte parmi les membres les plus actifs avec le baron du Havelt, dont les analyses sont essentiellement financières. Jusqu'à sa mort en 1856, le R.-P. Martin, sur lequel une étude complète dépassant les limites de cet article, devait être publiée, s'est battu pour imposer la forme antique ou semi-antique dans les commandes financées par l'administration des Cultes. Force est de constater qu'il n'y parvint guère puisqu'uniquement trente-cinq pontificaux décrits dans les documents, comme médiévaux, semi-médiévaux ou avec des décors influencés par l'art du Moyen Age, ont été recensés pendant la période concordataire. Prenant pour contre-exemples les prélats qui ont adopté avec ardeur le style antique, tels que Mgr de Dreux-Brézé, Mgr Nanquette, Mgr Sibour, l'érudit jésuite tente d'imposer le retour aux formes et aux décors du Moyen Âge. Il oriente sa critique vers le bougran, qu'il condamne parce qu'il donne aux vêtements des formes disgracieuses et raides, vers les étoffes brochées, comme on le voit dans le dossier du pontifical commandé pour la fabrique de la cathédrale de Châlons en 1852 à l'Ouvroir des Orphelines de la paroisse Notre-Dame de

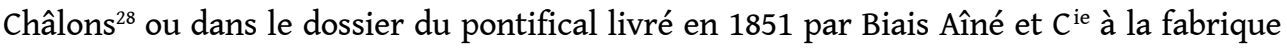


de la cathédrale de Périgueux ${ }^{29}$. Il critique la mauvaise qualité des dessins et il s'en plaint à plusieurs reprises dans ses rapports, notamment dans celui rédigé pour le pontifical commandé par la fabrique de la cathédrale de Fréjus en 1852 à Marteau et $C^{\text {ie }}:$ «Il est malheureusement trop vrai que la plupart des dessinateurs de nos fabriques les plus célèbres ne connaissent pas d'autre art que celui de la mode ${ }^{30}$.»Cette préoccupation est récurrente, on la retrouve dans une lettre qu'il adresse au ministre des Cultes le 4 novembre 1853 à propos d'un pontifical livré par $\mathrm{V}^{\text {ve }}$ Monteilhet, de Lyon à la fabrique de la cathédrale de Belley ${ }^{31}$. Il met en exergue les fournitures des églises «restées depuis trop longtemps entre les mains de la pure industrie ». Afin que l'administration impose ce qu'il appelle le bon goût, Arthur Martin encourage une production parisienne et en particulier celle de son ami Hubert Ménage. Il y parviendra sur quelques dossiers: le pontifical initialement commandé à $\mathrm{V}^{\mathrm{ve}}$ Granoux, de Digne pour la fabrique de la cathédrale de la même ville en 1852, sera réalisé par Hubert Ménage, permettant d'éviter qu'un goût déplorable ne s'éternise dans l' "ameublement ecclésiastique $»^{32}$. Bien que soutenu par l'administration des Cultes, le R.-P. Martin aura toutefois du mal à imposer son opinion. Sans doute dans un souci d'apaisement, l'administration des Cultes suit, dans la majorité des dossiers traités par la commission des arts et édifices religieux, l'opinion de l'ecclésiastique. S'il se montre réticent soit par goût soit parce que l'introduction du style médiéval dans une sacristie classique serait susceptible de choquer les fidèles, l'administration des Cultes ne lui refuse pas le financement demandé. C'est par exemple le cas à Limoges en 1852 pour le pontifical livré par Bent. L'influence de la commission des arts et édifices religieux pour la section ornements liturgiques fut donc limitée pendant son existence. Elle n'a toutefois, à sa décharge, examiné qu'une vingtaine de dossiers sur les cinq cent soixante-sept dossiers soumis à l'administration des Cultes pendant les années concordataires, soit un peu moins de 3,5\%. On peut aussi s'étonner que l'administration des Cultes n'ait que partiellement financé les pontificaux de forme médiévale qui lui étaient soumis. Par exemple, le pontifical en drap d'or confectionné par Hubert Ménage en 1857 pour la cathédrale de Saint-Claude, d'un montant de 5000 francs, de forme médiévale, ne bénéficie que de 3000 francs de financement public ${ }^{33}$. Il semble par ailleurs qu'un nombre non négligeable de prélats aient été fort réticents à l'introduction des formes et des décors médiévaux. L'affaire des dais de Viollet-le-Duc qui éclate dans les années 1850 constitue l'illustration la plus significative de ce rejet par la province et, et les simples fidèles, du mouvement néo-gothique pourtant vénéré et admiré par les intellectuels parisiens ${ }^{34}$. Plutôt qu'un retour aux sources médiévales et pures de la liturgie, les prélats recherchent d'avantage le faste et une liturgie propice à impressionner les fidèles. Pourtant, à partir des années 1865, l'influence d'Arthur Martin se révèle significative. Plusieurs pontificaux, confectionnés par Hubert Ménage sont totalement financés par l'administration des Cultes à la fin du Second Empire et surtout sous la III République, montrant l'évolution du goût vers un néogothique moins rigoureux et plus ornemental.

19 L'autre facteur susceptible d'influencer la production de pontificaux tient dans l'adoption progressive à partir des années 1850 de la liturgie romaine, qui va donner lieu à plusieurs demandes d'achats ou de confection de pontificaux. Les arguments avancés par les fabriques et les prélats sont de trois ordres: la couleur constitue le premier et, en particulier, les pontificaux de couleur blanche, comme le pontifical blanc en soie broché or fin devenu «indispensable pour les grandes solennités " depuis l'adoption du rite romain par l'évêque de Soissons ${ }^{35}$. Le pontifical est livré par Biais aîné fils et Rondelet en 1868. Le second argument concerne les livres liturgiques et le troisième est relatif au 
nombre de pièces nécessaires à la célébration du culte selon les règles de la liturgie romaine. Mgr de Dreux-Brézé justifie ainsi sa demande, datée du 3 mai 1853, de complément d'un pontifical blanc fourni par Hubert Ménage en $1852^{36}$. Les fabriques de Nantes, Bourges, Luçon, Coutances, Belley avancent les mêmes demandes dès la fin des années 1850.

Toutefois au-delà des requêtes justifiées par un changement de rite, nous n'avons pu démontrer l'existence d'un lien entre l'octroi d'une subvention pour l'achat d'un pontifical et les opinions ultramontaines ou gallicanes du prélat demandeur, le lien politique étant prédominant.

21 Le Second Empire constitue une période faste pour la production de pontificaux. Ces années révèlent combien le pontifical constitue un outil politique, il représente la présence du gouvernement dans la cathédrale, il affiche l'harmonie des relations entre l'Église et l'État, il participe au faste de la liturgie et au spectacle de la messe. Le Second Empire a mené une politique en faveur des formes gothiques, mais elle l'a conduite mollement, presque sans conviction, ne souhaitant pas s'opposer aux prélats réticents, ni empiéter sur un domaine qui devait rester sous leur direction. La commission des arts et édifices religieux ainsi que le R.-P. Martin, ont plus semé pour l'avenir que réellement récolté le fruit de leurs efforts. Peut-être était-ce trop tôt, les prélats comme les fidèles ne pouvant, avant la fin des années 1860 , accepter un tel retour aux formes du XIII siècle, souvent assimilé à un choix ou un goût purement parisien. Les pontificaux généralement en drap d'or ou en drap d'argent, surchargés de broderies, au dessin identique et répétitif, réalisées en guipure ou en gaufrure de fils d'or ou d'argent sur carton et appliquées, qui seront tant détestées quelques décennies plus tard, sont caractéristiques des réalisations du Second Empire. Leur richesse, voire leur clinquant, ont contribué au faste de la messe que décrit avec précision Zola à l'occasion du mariage de l'héroïne du Rêve $e^{37}$.

\section{NOTES}

1. Élisabeth Hardouin-Fugier, «Les commandes gouvernementales de vêtements pontificaux. 1820-1880 », Paramentica, Lyon, 1992, 197 p., p. 75-89.

2. Jean-Michel Leniaud, L'Administration des Cultes, Paris, 1988, 428 p., p. 99-100.

3. Arch. dioc. de Blois, 19N2B, dossier Cathédrale, Note adressée par le Garde des sceaux, ministre de la justice et des Cultes, Persil, aux préfets du 20 décembre 1841, circulaire $\mathrm{n}^{\circ} 55$.

4. Arch. nat., $\mathrm{F}^{19} 3830$, liasse Nancy, lettre du ministre des cultes à l'évêque de Nancy du $1^{\mathrm{er}}$ août 1863.

5. Arch. nat., $\mathrm{F}^{19}$ 3820, liasse Ajaccio, lettre de l'évêque au préfet de Corse du 2 avril 1855.

6. Ibid., Lettre du préfet de Corse au ministre des cultes du 22 novembre 1855.

7. Arch. nat., $\mathrm{F}^{19} 3829$, liasse Marseille, lettre au préfet des Bouches-du-Rhône au ministre des cultes pour transmission au 8 février 1852, Lettre du ministre des cultes au préfet des Bouchesdu-Rhône du 18 septembre 1852.

8. Arch. nat., $\mathrm{F}^{19} 3824$, liasse Blois.

9. Jean Maurain, La Politique ecclésiastique sous le Second Empire de 1852 à 1869, Paris, F. Alcan, 1930, 257 p., p. 313. 
10. Arch. nat., $\mathrm{F}^{19} 3828$, liasse Le Mans.

11. Arch. nat., $\mathrm{F}^{19} 3828$, liasse Luçon.

12. Arch. nat., $F^{19} 3827$, liasse Gap.

13. Arch. nat., $\mathrm{F}^{19} 3821$, liasse Amiens.

14. Arch. nat., $\mathrm{F}^{19} 3821$, liasse Alger, lettre du ministre de la justice et des cultes du 4 mai 1867 approuvant la dépense pour ornements divers passée à Caroline Bourscheidt; lettre du ministre de la justice et des cultes du 6 janvier 1869 approuvant la dépense pour un ornement en drap d'or passée à Caroline Bourscheidt, domiciliée à Nancy.

15. Arch. nat., $\mathrm{O}^{5} 420$ à $\mathrm{O}^{5} 438$.

16. Arch. nat., $\mathrm{F}^{19} 3836$, liasse Verdun.

17. Arch. nat., $\mathrm{F}^{19} 3820$, liasse Ajaccio.

18. Arch. nat., $\mathrm{F}^{19} 3834$, liasse Saint-Jean-de-Maurienne.

19. Arch. nat., $\mathrm{F}^{19} 3830$, liasse Nevers.

20. Arch. nat., $\mathrm{F}^{19}$ 3820, liasse Ajaccio.

21. Arch. nat., $\mathrm{F}^{19} 3828$, liasse Le Mans, le devis se monte à 9450 francs, la chape principale coûte 1200 francs, les chapes secondaires, 800 francs, le grémial et l'épistolier à 150 francs et 500 francs.

22. Arch. nat., $\mathrm{F}^{19} 3828$, liasse Le Mans.

23. Arch. nat., $\mathrm{F}^{19} 3836$, liasse Toulouse.

24. Ibid.

25. Arch. nat., $\mathrm{F}^{19}$ 3832, liasse Périgueux, le devis se monte à 3875 francs.

26. Dupuis a une boutique au 66 rue des Saints-Pères à Paris à l'enseigne "À Saint Eloi ", dans Arch. nat., $\mathrm{F}^{19}$ 3832, liasse Périgueux.

27. Arch. nat., $\mathrm{F}^{19}$ 3821, liasse Amiens, devis du 20 juin 1867.

28. Arch. nat., $\mathrm{F}^{19}$ 3826, liasse Châlons, rapport du 10 juin 1852.

29. Arch. nat., $\mathrm{F}^{19}$ 3832, liasse Périgueux, rapport du 12 septembre 1851.

30. Arch. nat., $\mathrm{F}^{19}$ 3827, liasse Fréjus, rapport du 15 décembre 1851.

31. Arch. nat., $\mathrm{F}^{19} 3824$, liasse Belley.

32. Arch. nat., $\mathrm{F}^{19}$ 3826, liasse Digne, rapport du 9 mars 1852.

33. Arch. nat., $F^{19} 3834$, liasse Saint-Claude, devis du 17 juillet 1857, approbation le 3 août 1857 par lettre du ministre des cultes au préfet.

34. Élisabeth Hardouin-Fugier, op. cit. : La commande des dais à Viollet-le-Duc remonte à une demande formulée par Mgr Mazenod, évêque de Marseille. Les premiers projets datent de juillet 1851. Viollet-le-Duc confie la réalisation du dais à Bachelet, un des trois orfèvres avec lesquels il a l'habitude de travailler. L'accueil des dais à Marseille, Carcassonne et Montauban est plus que défavorable alors que leur réception à Paris avait été excellente. Les dais sont renvoyés à Paris et trouvent preneurs : Gap accepte le dais de Montauban, celui de Marseille est envoyé à Angoulême et celui de Carcassonne est envoyé à Arras.

35. Arch. nat., $\mathrm{F}^{19}$ 3835, liasse Soissons, Délibération du conseil de la fabrique de Soissons en 1868.

36. Arch. nat., $\mathrm{F}^{19}$ 3830, liasse Moulins.

37. Émile Zola, Le Rêve, Paris, Livre de poche, 1985, 318 p., p. 181-184. 


\section{RÉSUMÉS}

Avec le Concordat s'est mis en place un système de subventions publiques octroyées par l'Administration des Cultes en faveur de l'achat, par les fabriques des cathédrales, de pontificaux. Le Second Empire a mené une politique particulièrement active dans ce domaine et y a consacré un budget important. Le pontifical devient dès lors un objet administratif, même si le Second Empire ne fait qu'appliquer des règles édictées par les gouvernements précédents, mais aussi un outil politique autant qu'esthétique.

With the Concordat agreement, a system of public subsidies was established, granted by the Administration des Cultes (Religious Worship Authority), to help cathedral councils to purchase pontificals. The Second Empire carried out an active policy in this field, which was allocated a generous budget. From then on, the pontificals became both administrative items, even if the Second Empire was simply applying the rules set up by previous governments, and political tools, as much as aesthetic ones.

Mit dem Konkordat wurden den fabriques des cathédrales (kirchliche Verwaltungsinstitutionen ) öffentliche Zuschüsse vom Ministère des Cultes gewährt, um besondere festliche Messgewänder, im französischen pontificaux, zu kaufen, die von da an als verwaltungsmäßige Gegenstände angesehen wurden. Das Second Empire, das sich dabei zwar an die Vorschriften der früheren Regierungen hielt, schlug aber eine besonders aktive Politik in diese Richtung ein. Es steckte beträchtliche Geldsummen in den Kauf von pontificaux, so dass diese Gewänder ebenso an politischer wie an ästhetischer Bedeutung gewannen.

\section{AUTEUR}

\section{MARIA-ANNE PRIVAT-SAVIGNY}

Maria-Anne Privat-Savigny est née en 1970 et diplômée de l'École supérieure des sciences économiques et commerciales (ESSEC, 1993), de l'École du Louvre (1994), de l'École nationale du patrimoine (1998), titulaire d'un DEA à l'université Paris VIII-Saint-Denis, Institut d'études européennes (Gestion et exploitation commerciale des musées dans quatre pays d'Europe : La France, l'Angleterre, l'Allemagne et l'Italie, étude comparée, 1995) et d'un DEA à l'École pratique des hautes études ( $«$ La reconstruction des cathédrales de Valence, Die et Saint-Paul-TroisChâteaux et premier inventaire des destructions et reconstructions d'édifices romans dans le midi de la France ", 1997). Elle est doctorante sous la direction de J.-M. Leniaud à l'École pratique des hautes études (Splendeur du Culte et gloire du gouvernement. L'administration des Cultes et les pontificaux pendant la période concordataire). Elle a été conservateur du patrimoine au sein du centre de Recherche et de restauration des Musées de France (section arts décoratifs, 1998-2001), conservateur au musée national de la Renaissance où elle a travaille sur les broderies au petit point de la fin $\mathrm{du} \mathrm{XVI}^{\mathrm{e}}$ siècle, sur la garniture de lit au XVI ${ }^{\mathrm{e}}$ siècle, tout en préparant le catalogue sur les broderies et textiles des collections du musée (2001-2004). Elle est conservateur 
au musée des Tissus de Lyon en 2005, puis directeur du musée des Tissus et du musée des arts décoratifs de Lyon depuis juin 2006, conservateur en chef du patrimoine depuis juin 2008. 